\title{
Spin Injection and Detection in Silicon
}

\author{
Igor Žutić, ${ }^{1,2}$ Jaroslav Fabian, ${ }^{3}$ and Steven C. Erwin ${ }^{2}$ \\ ${ }^{1}$ Department of Physics, State University of New York at Buffalo, Buffalo, New York 14260, USA \\ ${ }^{2}$ Center for Computational Materials Science, Naval Research Laboratory, Washington, DC 20375, USA \\ ${ }^{3}$ Institute for Theoretical Physics, University of Regensburg, 93040 Regensburg, Germany \\ (Received 21 December 2004; revised manuscript received 4 May 2006; published 14 July 2006)
}

\begin{abstract}
Spin injection and detection in silicon is a difficult problem, in part because the weak spin-orbit coupling and indirect gap preclude using standard optical techniques. Two ways to overcome this difficulty are proposed, both based on spin-polarized transport across a heterojunction. Using a realistic transport model incorporating the relevant spin dynamics of both electrons and holes, it is argued that symmetry properties of the charge current can be exploited to detect electrical spin injection in silicon using currently available techniques.
\end{abstract}

DOI: 10.1103/PhysRevLett.97.026602

PACS numbers: 72.25.Dc, 72.25.Mk

In addition to its central role in conventional electronics, silicon has spin-dependent properties (such as long spinrelaxation and decoherence times) that could be particularly useful in spin-based quantum-information processing and spintronics [1]. Unfortunately, the underlying origins of these attractive properties - the indirect band gap, weak spin-orbit coupling, and small concentration of paramagnetic impurities [2] -also preclude using the standard optical methods of spin injection and detection in semiconductors. Robust spin injection and detection in silicon has already been pursued for decades [1]. Some schemes use (ferromagnetic) metal-Si hybrid structures for electrical injection or detection [3] or suggest magnetic sensitivity of photoresponse during light illumination [4]. However, material difficulties, such as the formation of silicides and interface quality, are compounded by the lack of a theoretical description of nonequilibrium spin transport that accounts for the complexity of such inhomogeneous hybrid structures. Consequently, it remains a challenge to quantify the degree to which spurious effects and magnetoresistance would compete with signals that might be attributed to spin injection.

Circularly polarized light can be used to polarize carriers in semiconductors with a direct band gap. Moreover, both the direction and the magnitude of optically generated charge currents [5] and pure spin currents [6] can be controlled optically. In the reverse process, the presence of polarized carriers in a direct-gap semiconductor can be detected by measuring the circular polarization of the recombination light [1,7]. Typical detection schemes use spin light-emitting diodes (LEDs), where the selection rules for radiative recombination processes can be used to relate the circular polarization of the emitted light to the spin polarization of the carriers [8].

For silicon, the indirect band gap makes direct application of these techniques problematic. Here we propose two approaches to overcome this difficulty. The first, shown in Fig. 1(a), is based on high-quality heterojunctions between $\mathrm{Si}$ and a direct-gap semiconductor. In such a heterojunction, optical techniques could be readily employed in the direct-gap semiconductor to circumvent the problems with spin injection and detection in Si. The key prerequisite for such a proposal is an interface with Si that would not be detrimental to the spin transport. This is a nontrivial undertaking, as the lattice mismatch between $\mathrm{Si}$ and most directgap semiconductors typically leads to low-quality interfaces with a high density of interfacial defects. Nevertheless, there has been recent progress in fabricating high-quality $\mathrm{GaAs} / \mathrm{Si}$ interfaces (despite the 4\% lattice mismatch) [9]; $\mathrm{GaP}_{1-x} \mathrm{~N}_{x}$ leads to even smaller mismatch (below 1\%) [10]. Recent studies of charge transport in GaAs/Si heterojunctions as well as optical excitations in GaAs LEDs grown on $\mathrm{Si}$ [11] suggest the feasibility of the scheme shown in Fig. 1(a). In particular, GaAs/Si heterojunctions displayed $I-V$ characteristics of an ideal diode [12].

In the second approach, shown in Fig. 1(b), magnetic semiconductors approximately lattice matched with $\mathrm{Si}$ could be used for spin injection and detection [13]. For example, the $\mathrm{Mn}$-doped chalcopyrite $\mathrm{ZnGeP}_{2}$ (mismatch

(a)
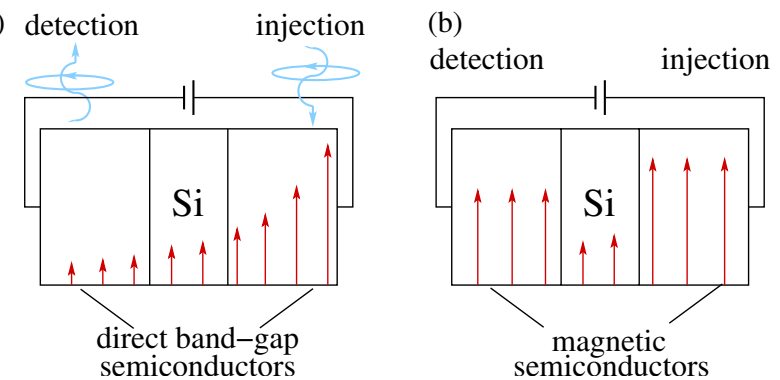

FIG. 1 (color online). Proposed schemes for spin injection and detection in silicon. (a) Optical realization based on radiative processes in direct-gap semiconductors surrounding silicon. Arrows depict the spatial decay of nonequilibrium spin. (b) Electrical realization based on the spin splitting and the net spin density (magnetization) in the two magnetic regions. The relative orientation of the nonequilibrium spin in $\mathrm{Si}$ and the equilibrium spin in the magnetic regions influences the magnitude of a charge current or an open-circuit voltage. Other realizations are also possible by combining schemes (a) and (b). 
$<2 \%$ ) [14] has been reported to be ferromagnetic at room temperature. Another Mn-doped chalcopyrite, $\mathrm{ZnSiP}_{2}$, was recently predicted [15] to be ferromagnetic, as well as highly spin polarized and closely lattice-matched with $\mathrm{Si}$ (mismatch $<1 \%$ ). Mn doping of the chalcopyrite alloy $\mathrm{ZnGe}_{1-x} \mathrm{Si}_{x} \mathrm{P}_{2}$ could lead to an exact match.

To capture the main features of spin-polarized transport across a heterojunction we formulate here a model, representing both schemes in Fig. 1. One can model the right (injecting) electrode by appropriate boundary conditions, and we focus on the two left regions that in our model define the heterojunction, doped with ionized acceptors and donors of density $N_{a}$ and $N_{d}$. Codoping with magnetic impurities would additionally introduce a net spin, but need not change the number of carriers. An inhomogeneous distribution of $N_{a, d}$ implies a large deviation from local charge neutrality and Poisson's equation must be explicitly solved. For nondegenerate doping the spin-resolved quasiequilibrium electron and hole densities are

$$
n_{\lambda}=\frac{N_{c}}{2} e^{-\left[E_{c \lambda}-\mu_{n \lambda}\right] / k_{B} T}, \quad p_{\lambda}=\frac{N_{v}}{2} e^{-\left[\mu_{p \lambda}-E_{v \lambda}\right] / k_{B} T},
$$

where $\lambda=+1$ for spin up $(\uparrow)$ and -1 for spin down $(\downarrow)$. The total electron density $n=n_{\uparrow}+n_{\downarrow}$ can also be decomposed as a sum of equilibrium and nonequilibrium parts, $n=n_{0}+\delta n$. We define the electron-spin density $s_{n}=$ $n_{\uparrow}-n_{\downarrow}$ and the spin polarization $P_{n}=s_{n} / n$, with an analogous notation for holes. In Eq. (1), subscripts $c$ and $v$ label quantities pertaining to conduction and valence bands. The corresponding effective density of states are $N_{c, v}=2\left(2 \pi m_{c, v} k_{B} T / h^{2}\right)^{3 / 2}$, where $m_{c, v}$ are effective masses. The spin- $\lambda$ conduction band edge (see Fig. 2) $E_{c \lambda}=E_{c 0}-q \phi-\lambda q \zeta_{c}$ differs from its nonmagnetic bulk value $E_{c 0}$ because of the electrostatic potential $\phi$ and the spin splitting $2 q \zeta_{c}$, which parametrizes Zeeman or exchange splitting due to magnetic impurities and/or an applied magnetic field [1]. Here, $\Delta E_{c}$ is the conductionband-edge discontinuity and $\mu_{n \lambda}=\mu_{0}+\delta \mu_{n \lambda}$ is the chemical potential for spin- $\lambda$ electrons. An analogous notation holds for the valence band and holes.

We assume that transport is dominated by drift diffusion, so that the spin-resolved current densities are

$$
\begin{gathered}
\mathbf{J}_{n \lambda}=\bar{\mu}_{n \lambda} n_{\lambda} \nabla E_{c \lambda}+q D_{n \lambda} N_{c} \nabla\left(n_{\lambda} / N_{c}\right), \\
\mathbf{J}_{p \lambda}=\bar{\mu}_{p \lambda} p_{\lambda} \nabla E_{v \lambda}-q D_{p \lambda} N_{v} \nabla\left(p_{\lambda} / N_{v}\right),
\end{gathered}
$$

where $\bar{\mu}$ and $D$ are mobility and diffusion coefficients. "Drift terms" have quasielectric fields $\propto \nabla E_{c, v \lambda}$ that are generally spin dependent $\left[\nabla \zeta_{c, v} \neq 0\right.$ is referred to as a magnetic drift [16]] and different for conduction and valence bands. In contrast to homojunctions, additional "diffusive terms" arise due to the spatial dependence of $m_{c, v}$ and $N_{c, v}$.

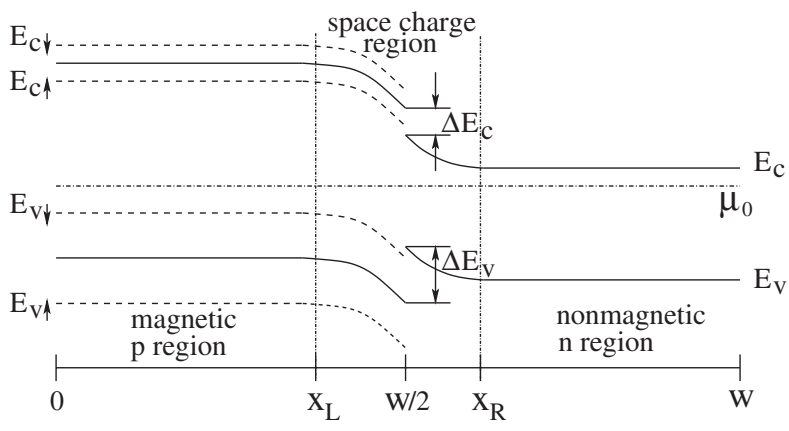

FIG. 2. Band diagram for a magnetic heterojunction. In equilibrium, the chemical potential $\mu_{0}$ is constant. Conduction- and valence-band edges $\left(E_{c, v}\right)$ are spin split in the magnetic $p$ region, while there is no spin splitting in the nonmagnetic $n$ region (corresponding to $\mathrm{Si}$ ). For a sharp doping profile, there are generally band-edge discontinuities $\left(\Delta E_{c, v}\right)$ at $x=w / 2$.

We write the continuity equation for $n_{\lambda}$ as

$$
\begin{aligned}
-\partial n_{\lambda} / \partial t+\nabla \cdot \mathbf{J}_{n \lambda} / q= & +r_{\lambda}\left(n_{\lambda} p_{\lambda}-n_{\lambda 0} p_{\lambda 0}\right) \\
& +\left[n_{\lambda}-n_{-\lambda}-\lambda P_{n 0} n\right] / 2 \tau_{\mathrm{sn}}-G_{\lambda},
\end{aligned}
$$

with an analogous equation for $p_{\lambda}$. Here, $r_{\lambda}$ is the coefficient of the recombination rate of spin- $\lambda$ carriers, $\tau_{\mathrm{sn}, p}$ is the spin-relaxation time for electrons and holes, and $G_{\lambda}$ is the photoexcitation rate due to electron-hole pair generation and optical orientation (when $G_{\uparrow} \neq G_{\downarrow}$ ) [1,7]. Spin relaxation equilibrates carrier spin while preserving nonequilibrium carrier density [7], so that for nondegenerate semiconductors we have $P_{n 0}=\tanh \left(q \zeta_{c} / k_{B} T\right)$.

We make several assumptions to solve this model analytically. We focus here on the steady-state low-injection regime, at applied bias $|V|<\min \left(\left|E_{c \lambda}-E_{v \lambda}\right|\right)$. Spin-orbit coupling in the valence band typically leads to a much faster spin-relaxation of holes than electrons [3-4 orders faster in GaAs [1] ], and so it is reasonable to consider that the hole spins are in equilibrium $\left(\delta s_{p}=0\right.$ and $\left.P_{p}=P_{p 0}\right)$. As a result, only the nonequilibrium electron-spin density $\left(\delta s_{n} \rightarrow \delta s\right)$ and the minority carrier density need to be calculated throughout the heterojunction. We assume a sharp doping profile $N_{d}(x)-N_{a}(x)$; referring to Fig. 2, this leads to a discontinuous change in materials parameters at $x=w / 2$. We take $N_{c, v}, \bar{\mu}, D$, and the permittivity $\epsilon$ to be constant outside the space-charge region $x_{L}<$ $x<x_{R}$, and hence label them by indices $L$ and $R$. The width of a space-charge region is $x_{R}-x_{L} \propto\left(V_{b i}-V\right)^{1 / 2}$, where the built-in voltage is $q V_{b i}=-\Delta E_{c}+$ $k_{B} T \ln \left(n_{0 R} N_{c R} / n_{0 L} N_{c L}\right)$. The discontinuity $\Delta E_{c, v}$ can be accurately measured at interfaces with $\mathrm{Si}$ [17]. Equations (2) and (3), together with the continuity equations, reduce to diffusionlike equations for $\delta n, \delta s$ in the $p$ region and $\delta p, \delta s$ in the $n$ region. For the (magnetic) $p$ region, we find that the spatial dependence of both $\delta n$ and $\delta s$ are described by two distinct decay lengths; this is in marked contrast to previously studied cases [1]. These 
decay lengths can be written as

$$
\begin{aligned}
\kappa^{-1}, \chi^{-1}= & \left\{\left(L_{\uparrow}^{-2}+L_{\downarrow}^{-2}+L_{s}^{-2}\right) / 2 \pm\left[\left(L_{\uparrow}^{-2}-L_{\downarrow}^{-2}\right.\right.\right. \\
& \left.\left.\left.-P_{n 0} L_{s}^{-2}\right)^{2}+\left(1-P_{n 0}^{2}\right) L_{s}^{-4}\right]^{1 / 2} / 2\right\}^{-1 / 2},
\end{aligned}
$$

where the upper sign refers to $\kappa^{-1}, L_{\lambda}=\left(D_{n} / r_{\lambda} p_{\lambda 0}\right)^{1 / 2}$ are the electron diffusion lengths, and $L_{s}=\left(D_{n} \tau_{\mathrm{sn}}\right)^{1 / 2}$ is the electron-spin diffusion length.

It is instructive to consider the regime of spinunpolarized holes, appropriate for the scheme shown in Fig. 1(a). In this regime we have $p_{\uparrow 0}=p_{\downarrow 0}=N_{a} / 2$ and $r_{\uparrow}=r_{\downarrow}=2 r$ [in a nondegenerate regime $r_{\uparrow} \approx r_{\downarrow}$ even for $P_{p} \neq 0$ [18] ]. It follows from Eq. (5) that $\kappa^{-1}$ reduces to $L_{\mathrm{sn}}=\left(D_{n} T_{s}\right)^{1 / 2}$, where $L_{\mathrm{sn}}$ is the effective electron-spin diffusion length and $T_{s}=\left(r N_{a}+1 / \tau_{\mathrm{sn}}\right)^{-1}$ is the electronspin lifetime. Analogously, $\chi^{-1}$ reduces to the electron diffusion length $L_{n}=\left(D_{n} \tau_{n}\right)^{1 / 2}$, where $\tau_{n}=1 / r N_{a}$ is the electron lifetime [7]. Thus, in this regime $\kappa^{-1}$ and $\chi^{-1}$ separately determine the decay lengths for $\delta s$ and $\delta n$, respectively. For the more general case, in Fig. 3 we show $\kappa^{-1}$ and $\chi^{-1}$ as a function of $P_{p}$. From the behavior of the decay lengths for $\delta n$ and $\delta s$, we conclude that polarization of the equilibrium hole spins leads to a strong modification of charge and spin dynamics of electrons.

We turn now to the more general case of spin detection using magnetic semiconductors, shown in Fig. 1(b), and solve the corresponding problem of spin-polarized transport across the heterojunction in Fig. 2. We impose the Ohmic boundary conditions $\delta n=\delta s=0$ at $x=0$, and include optical or electrical carrier and spin injection through the boundary conditions $\delta p \neq 0$ and $\delta s \neq 0$ at $x=w$. To match the chemical potentials $\mu_{n, p \lambda}$ at $x_{L}$ and $x_{R}$ requires satisfying the self-consistency condition $P_{n}^{L}=\left(P_{n 0}^{L}+\delta P_{n}^{R}\right) /\left(1+P_{n 0}^{L} \delta P_{n}^{R}\right)$, where $\delta P_{n}^{R}=\delta s_{R} / N_{d}$ is determined from the continuity of the spin current, $D_{n L} d\left(\delta s_{L}\right) / d x=D_{n R} d\left(\delta s_{R}\right) / d x$. Consistent with this matching, a generalization of Shockley's relation [19]

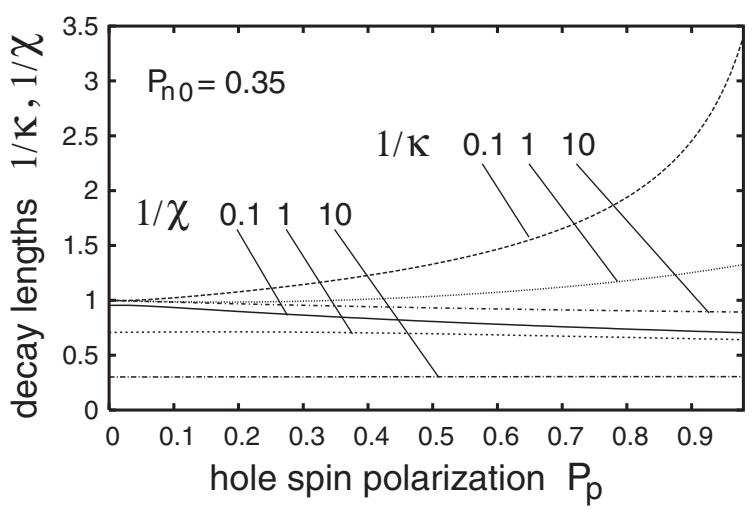

FIG. 3. Decay lengths $\kappa^{-1}$ and $\chi^{-1}$ in the magnetic $p$ region, normalized to the electron diffusion length $L_{n}=\left(D_{n} \tau_{n}\right)^{1 / 2}$, as a function of hole-spin polarization for fixed electron spin polarization $P_{n 0}=0.35$. Each curve is labeled by the ratio of electron lifetime to spin-relaxation time, $\tau_{n} / \tau_{s n}$. is $\delta n_{L}=n_{0 L}\left[\exp \left(q V / k_{B} T\right)-1\right]+s_{0 L} \exp \left(q V / k_{B} T\right) \delta P_{n}^{R}$ and $\delta s_{L}=s_{0 L}\left[\exp \left(q V / k_{B} T\right)-1\right]+n_{0 L} \exp \left(q V / k_{B} T\right) \delta P_{n}^{R}$, such that $\delta n_{L}, \delta s_{L}$ and $\delta n_{R}, \delta s_{R}$ can be considered as boundary conditions in the $p$ and $n$ region, respectively. For the $p$ region we then obtain

$$
\begin{aligned}
\delta n, \delta s= & -C_{\kappa n, s} \frac{\left(\chi^{2}+a\right) \delta n_{L}+b \delta s_{L}}{\left(\kappa^{2}-\chi^{2}\right) \sinh \left(\kappa x_{L}\right)} \sinh (\kappa x) \\
& +C_{\chi n, s} \frac{\left(\kappa^{2}+a\right) \delta n_{L}+b \delta s_{L}}{\left(\kappa^{2}-\chi^{2}\right) \sinh \left(\chi x_{L}\right)} \sinh (\chi x),
\end{aligned}
$$

where $C_{\kappa n}=C_{\chi n}=1$ for $\delta n, C_{\kappa s}=-\left(\kappa^{2}+a\right) / b$ and $C_{\chi s}=-\left(\chi^{2}+a\right) / b$ for $\delta s$, and we have defined $a=$ $-\left(L_{\uparrow}^{-2}+L_{\downarrow}^{-2}\right) / 2$ and $b=-\left(L_{\uparrow}^{-2}-L_{\downarrow}^{-2}\right) / 2$.

In the $n$ region (representing $\mathrm{Si}$ ), the total charge current $J$ is the sum of the minority carrier currents at $x_{L}$ and $x_{R}$, $J=J_{n L}+J_{p R}$, in analogy to Shockley's formulation [20]. $J_{n L}=q D_{n L} d\left(\delta n_{L}\right) / d x$, and $d\left(\delta n_{L}\right) / d x$ can be evaluated using Eq. (6). A straightforward method for detecting injected spin in Si follows from the symmetry properties of the different contributions to the charge current under magnetization reversal. By reversing the equilibrium spin polarization using a modest external magnetic field $\left(P_{n 0}\right.$, $\left.P_{p} \rightarrow-P_{n 0},-P_{p}\right)$ it follows from Eq. (5) that $\kappa, \chi \rightarrow \kappa$, $\chi$ and $a, b \rightarrow a,-b$. A part of $J_{n L}$, odd under such reversal, can be identified as the spin-voltaic current [16],

$$
J_{s v} \propto q D_{n L} n_{0 L} \exp \left(q V / k_{B} T\right) \delta P_{n}^{R} /\left(\kappa^{2}-\chi^{2}\right),
$$

which originates from the interplay of the equilibrium and nonequilibrium (injected) spin polarization in the $p$ and the $n$ region, respectively. Measurements of $J\left(V, P_{n 0}, P_{p}\right)-J\left(V,-P_{n 0},-P_{p}\right)=2 J_{s v}\left(V, P_{n 0}, P_{p}\right)$ would then provide: (1) cancellation of contributions to the charge current that are not related to the injected spin in Si and (2) a choice of $V$ to facilitate a sufficiently large $J_{s v}$ for accurate detection. Alternatively, for the optical detection scheme of Fig. 1(a), one would consider the limit $P_{n 0}=P_{p}=0$ in Eq. (6), and evaluate $P_{n}(x)$.

We illustrate the effects of spin injection and detection in $\mathrm{Si}$ using the heterojunction in Fig. 2. Figure 4 shows both the analytical results discussed above and the corresponding numerical results from the self-consistent solution of Poisson's equation and the four drift-diffusion equations [Eqs. (2)-(4)]. Numerical results require only imposing boundary conditions at $x=0, w$ and no assumptions about matching conditions at $x_{L, R}$. We use a standard set of parameters for $\mathrm{Si}$ doped with $N_{d}=10^{17} \mathrm{~cm}^{-3}$ [21]: $N_{c R}=3.2 \times 10^{19} \mathrm{~cm}^{-3}, D_{n R}=4 D_{p R}=20 \mathrm{~cm}^{2} / \mathrm{s}, \epsilon_{R}=$ $11.7, \tau_{p}=10^{-7} \mathrm{~s}$, an intrinsic carrier density [19] $n_{i R}=$ $10^{10} \mathrm{~cm}^{-3}$, and estimated spin-relaxation time in $\mathrm{Si}$ as $\tau_{s R}=10^{-7} \mathrm{~s}$. Circularly polarized light in zinc blende semiconductors, such as GaAs, generates spin polarization of up to $\delta P_{n}=0.5$, which can be increased even further using strain or quantum confinement $[1,7]$. We model the effects of spin injection from a neighboring direct-gap semiconductor, as depicted in Fig. 1, by assum- 


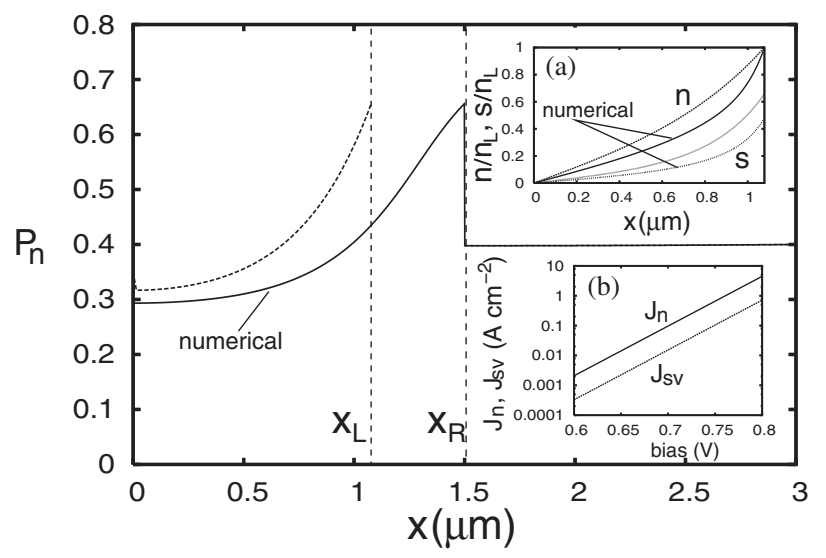

FIG. 4. Electron-spin polarization, $P_{n}$, for a magnetic heterojunction at forward bias $V=0.8 \mathrm{~V}$. The equilibrium spin polarizations in the $p$ region are $P_{n 0}=0.35$ and $P_{p}=0.8$, while the injected spin polarization in the $n$ region is $\delta P_{n}=0.4$ at $w=$ $3 \mu \mathrm{m}$. For $x>x_{R}$ numerical and analytical results coincide. Inset (a) profile of the electron carrier and spin density in the $p$ region, normalized to the corresponding electron density at $x_{L}=1.078 \mu \mathrm{m}(V=0.8 \mathrm{~V})$. Inset (b) voltage dependence of the electron charge current $J_{n}$ and its part due to the nonequilibrium spin (the spin-voltaic current $J_{s v}$ ).

ing $\delta P_{n}=0.4$ at $x=w$, and we set $\Delta E_{c}=0.2$. For a ferromagnetic semiconductor in the $p$ region we choose $N_{a}=10^{15} \mathrm{~cm}^{-3}, N_{c L}=10^{19} \mathrm{~cm}^{-3}, D_{n L}=5 \mathrm{~cm}^{-3} / \mathrm{s}, \epsilon_{L}=$ $10, \tau_{n}=10^{-9} \mathrm{~s}, \quad n_{i L}=(0.6)^{1 / 2} 10^{6} \mathrm{~cm}^{-3}$, and $\tau_{s L}=$ $10^{-10} \mathrm{~s}$. We parametrize the spin splitting of carrier bands (see Fig. 2) with $P_{n 0}=0.35$ and $P_{p}=0.8$ which, for Boltzmann statistics, corresponds to a splitting of $q \zeta_{v} \approx$ $3 q \zeta_{c} \approx k_{B} T$ [22]. The main plot in Fig. 4 shows $P_{n}(x)$. In the lightly doped $p$ region there is a deviation from the numerical result which can be attributed to drift effects [1] even for $x<x_{L}$ and the accuracy of the assumption of matching chemical potentials at $x_{L, R}$. However, at various biases, analytical results accurately give the maximum value for $P_{n}$ and, as can been seen for $x>x_{R}$ numerical and analytical solutions coincide. The effect of decay lengths in the $p$ region (analytic results yield $\kappa^{-1} \approx$ $0.22 \mu \mathrm{m}$ and $\left.\chi^{-1} \approx 0.64 \mu \mathrm{m}\right)$ can be seen in the Fig. 4(a). Analogous to $P_{n}(x)$, the decay of $n, s$ is influenced by drift effects and numerical and analytical results differ slightly. In the $n$ region, $L_{s R}=\left(D_{n R} \tau_{s R}\right)^{1 / 2} \approx 14 \mu \mathrm{m}$ implies a weak spin decay $\left(\delta P_{n}^{w} \approx \delta P_{n}^{R}\right)$, and the selfconsistency condition gives an estimate $P_{n}^{L} \approx(0.35+$ $0.4) /(1+0.35 \times 0.4) \approx 0.66$. We find a large "signal-tobackground ratio," $J_{s v} / J_{n}$, over a range of bias voltages. Analytical results, which do not require an additional numerical differentiation or current subtraction, are shown in Fig. 4(b). We thus predict that the magnitude of this spin-voltaic current - a fingerprint for spin injection and detection in $\mathrm{Si}$ - could readily be detected using existing experimental techniques [23].

Our theoretical framework could be also used to calculate the effects of nonequilibrium spin in other magnetic heterojunctions, including Si-based spin transistors [24]. An extension to the regime of large forward bias could provide an alternative method to detect nonequilibrium spin in Si. Even without a source of spin injection, a nonequilibrium spin could be generated through the process of spin extraction $[16,25]$ into a neighboring magnetic region. Our findings should also provide useful test cases for developing more general numerical methods for treating spin-polarized transport [26].

This work was supported by ONR, NSF-ECS CAREER, DARPA, NRC, SFB 689, CNMS and CCS at ORNL.

[1] I. Žutić et al., Rev. Mod. Phys. 76, 323 (2004).

[2] A. M. Tyryshkin et al., Phys. Rev. B 68, 193207 (2003).

[3] W. J. Wang et al., J. Magn. Magn. Mater. 272-276, 1915 (2004); E. Y. Tsymbal et al., Phys. Rev. B 66, 073201 (2002).

[4] V. K. Dugaev et al., Physica (Amsterdam) 16E, 558 (2003).

[5] S. D. Ganichev et al., Phys. Rev. Lett. 88, 057401 (2002); Nature (London) 417, 153 (2002).

[6] M. J. Stevens et al., Phys. Rev. Lett. 90, 136603 (2003); J. Hübner et al., Phys. Rev. Lett. 90, 216601 (2003).

[7] Optical Orientation, edited by F. Meier and B.P. Zakharchenya (North-Holland, Amsterdam, 1984).

[8] R. Fiederling et al., Nature (London) 402, 787 (1999); B. T. Jonker et al., Phys. Rev. B 62, 8180 (2000); D. K. Young et al., Appl. Phys. Lett. 80, 1598 (2002); X. Jiang et al., Phys. Rev. Lett. 90, 256603 (2003).

[9] H. Yonezu, Semicond. Sci. Technol. 17, 762 (2002).

[10] I. Vurgaftman et al., J. Appl. Phys. 89, 5815 (2001).

[11] P. J. Taylor et al., J. Appl. Phys. 89, 4365 (2001).

[12] E. Aperathitis et al., Appl. Surf. Sci. 102, 208 (1996).

[13] $\mathrm{Ga}_{1-x} \mathrm{Mn}_{x}$ As was already grown on $\mathrm{Si}$ [J. H. Zhao et al., J. Cryst. Growth 237-239, 1349 (2002)].

[14] Y. Ishida et al., Phys. Rev. Lett. 91, 107202 (2003); S. Cho et al., Phys. Rev. Lett. 88, 257203 (2002).

[15] S. C. Erwin and I. Žutić, Nature Mater. 3, 410 (2004).

[16] I. Žutić et al., Phys. Rev. Lett. 88, 066603 (2002).

[17] Yu. D. Glinka et al., Phys. Rev. B 65, 193103 (2002).

[18] N. Lebedeva et al., J. Appl. Phys. 93, 9845 (2003).

[19] J. Fabian et al., Phys. Rev. B 66, 165301 (2002).

[20] W. Shockley, Electrons and Holes in Semiconductors (Van Nostrand, Princeton, 1950).

[21] Handbook Series on Semiconductor Parameters, edited by M. Levinshtein et al. (World Scientific, Singapore, 1996).

[22] Even if, at present, such spin polarizations are difficult to achieve at room temperature, theoretical calculations [15] and high spin-polarization measurements at low temperature [J. G. Braden et al., Phys. Rev. Lett. 91, 056602 (2003); R. P. Panguluri et al., Phys. Rev. B 72, 054510 (2005)] suggest that similar values should be feasible in ferromagnetic semiconductors at $T \sim 100 \mathrm{~K}$.

[23] B. T. Jonker (private communication); T. Kondo et al., Jpn. J. Appl. Phys. 45, L663 (2006)

[24] C. L. Dennis et al., J. Magn. Magn. Mater. 290-291, 1383 (2005).

[25] A. M. Bratkovsky et al., J. Appl. Phys. 96, 4525 (2004).

[26] S. Saikin et al., J. Appl. Phys. 94, 1769 (2003). 